\title{
Additive Manufacturing and size-dependent mechanical properties of three-dimensional microarchitected, high-temperature ceramic metamaterials
}

\author{
Huachen Cui \\ Department of Mechanical Engineering, Virginia Tech, Blacksburg, Virginia 24061, USA \\ Ryan Hensleigh \\ Macromolecules Innovation Institute, Virginia Tech, Blacksburg, Virginia 24061, USA \\ Hongshun Chen \\ Department of Mechanical Engineering, Virginia Tech, Blacksburg, Virginia 24061, USA \\ Xiaoyu Zheng ${ }^{\text {a) }}$ \\ Department of Mechanical Engineering, Virginia Tech, Blacksburg, Virginia 24061, USA; and Macromolecules \\ Innovation Institute, Virginia Tech, Blacksburg, Virginia 24061, USA
}

(Received 2 October 2017; accepted 14 December 2017)

\begin{abstract}
3D microarchitected metamaterials exhibit unique, desirable properties influenced by their small length scales and architected layout, unachievable by their solid counterparts and random cellular configurations. However, few of them can be used in high-temperature applications, which could benefit significantly from their ultra-lightweight, ultrastiff properties. Existing high-temperature ceramic materials are often heavy and difficult to process into complex, microscale features. Inspired by this limitation, we fabricated polymer-derived ceramic metamaterials with controlled solid strut size varying from $10-\mu \mathrm{m}$ scale to a few millimeters with relative densities ranging from as low as 1 to $22 \%$. We found that these high-temperature architected ceramics of identical 3D topologies exhibit size-dependent strength influenced by both strut diameter and strut length. Weibull theory is utilized to map this dependency with varying single strut volumes. These observations demonstrate the structural benefits of increasing feature resolution in additive manufacturing of ceramic materials. Through capitalizing upon the reduction of unit strut volumes within the architecture, high-temperature ceramics could achieve high specific strength with only fraction of the weight of their solid counterparts.
\end{abstract}

\section{INTRODUCTION}

Engineering ceramics offer several beneficial properties, including high strength, wear resistance, high-temperature stability, and lightweight, compared to metals. These properties are desirable for many applications, including aerospace components such as gas turbine engines, ${ }^{1-5}$ as catalyst support, ${ }^{6}$ bioceramic scaffolds for tissue engineering, ${ }^{7,8}$ as temperature-resistant electronics, microelectromechanical systems, ${ }^{9-13}$ etc. In spite of these desirable properties, bulk ceramics have limited applications due to their high flaw sensitivity, bulky weight, and catastrophic fracture behavior upon loading, attributed to the persistence of distributed flaws within the ceramics: cracks, voids, and inclusions, ${ }^{14-16}$ which leads to a reduction in the fracture strength. Forming ceramics into highly complex shapes and interconnected porosities with high precision is, therefore, nearly impossible when

\footnotetext{
a) Address all correspondence to this author.

e-mail: raynexzheng@vt.edu

DOI: $10.1557 /$ jmr.2018.11
}

using traditional ceramic manufacturing and processing technologies.

Only a few fabrication approaches for accessing miniaturized ceramic features have been reported, which typically rely on additive manufacturing (AM) of ceramic particles in polymer resin followed by sintering or nanoscale coatings on top of a pre-made microstructure template. ${ }^{17,18}$ The former approach, AM with particleloaded polymers, allows for the fabrication of macro- and micro-lattices comprised of solid ceramic strut members. The particle process has major drawbacks including light scattering and limited loading of dispersed ceramic particles, which results in the final material likely with inevitable porosities and a large population of flaws. As such, the resolution and mechanical properties are significantly compromised. The template approach using nanoscale coating usually starts with AM-made polymer micro or nanolattices from projection microstereolithography or two-photon lithography. ${ }^{19-21}$ Atomic layer deposition (ALD), a highly conformal deposition process, is then used to coat uniform layers of ceramics with thickness control at the atomic level, producing composite 
microfeatures of polymer scaffold and nanoscale ceramic coating. The composite structures can be cut open for removal of the polymer template by etching or thermal treatment to leave behind hollow ceramic shell-like structures. ${ }^{22}$ The advantage of the templating approach is the uniform, conformal coating of ceramics and the nanometer thickness control of the ceramics. However, due to the nature of this templating approach, the resulting ceramics are only limited to a shell-like morphology, making it impossible to produce arbitrary microscale ceramic features. Additionally, the slow rate of the ALD process normally on the order of nanometers per hour makes scaling impractical. Glassy carbon lattices were previously fabricated by AM followed by a carbonization process, ${ }^{23,24}$ achieving a high strength-to-density ratio. However, carbon is not stable at high temperature in open atmosphere and hence has limited high-temperature applications.

New advances in AM of ceramics have taken advantage of ultraviolet (UV)-curable preceramic monomers, which upon heat treatment are directly converted into polymer-derived ceramics (PDCs) with virtually no porosity. ${ }^{25}$ Preceramic monomers rely on inorganic polymers such as carbosilane, siloxane, or silazane, bearing UV-curable active functional side groups such as thiols, vinyls, or epoxides. UV crosslinking during the $\mathrm{AM}$ processes converts these to net-shaped "green" preceramics which are then converted to a ceramic by inert atmosphere pyrolysis at high $\left(\sim 1000{ }^{\circ} \mathrm{C}\right)$ temperature, driving off volatile organic species (e.g., $\mathrm{H}_{2} \mathrm{O}$, $\mathrm{CO}_{2}$, and $\mathrm{CH}_{4}$ ). To date, complex-shaped cellular ceramic parts have been produced with virtually no defect porosity using commercially available $3 \mathrm{D}$ printers, achieving feature sizes in the range of hundreds of micrometers. ${ }^{25,26}$ Further reduction of the feature size and improved resolutions have not been reported and still present challenges. As the volatile organic species are driven off during the process, there is the potential for void formation and cracking in ligaments especially for thick sections. The pre-existing defects or flaws during the fabrication of the preceramic polymer lattice also affect the final quality of the 3D printed ceramic.

From a structural perspective, the benefit to reduce the controllable feature size in ceramics is the promise of incorporating size effects and precisely defined 3D architected topologies, which gives rise to the so-called new class of "architected metamaterials". Architected metamaterials are typically produced using the template ALD approach, motivated to attain nanoscale hollow shells. Through reducing the coating thickness, a 2-fold increase in strength was observed for the lower thickness compared to larger thickness as a result of the size effect. While the template ALD process allows for an indirect approach to capitalize on the size effects through reducing coating thickness, the most notable limitations are the challenge of rigorous control of structural integrity from the combined coating and post-etching process and the limited core-shell morphologies.

We seek to move beyond the limitations of the template ALD approach, by improving the resolution of PDC into the size effect strengthening regime. In this work, we describe the fabrication and mechanical properties of solid high-temperature architected metamaterials with microsized thickness and provide direct observation of the size-dependent mechanical properties as a result of the reduction of individual free surface ligament volume. These high-temperature PDCs are produced by highresolution large-area projection microstereolithography [Fig. 1(a)] with a resolution of several microns. The preceramic monomers are cured with near-UV light, forming 3D polymer structures that can have sub-10$\mu \mathrm{m}$ features [the gear teeth in Figs. 1(b) and 1(c)] and complex cellular architectures [Fig. 1(d)] spanning from $10-\mu \mathrm{m}$ scale to several centimeters. These polymer structures can be pyrolyzed to silicon oxycarbide (SiOC) components with uniform shrinkage and virtually no porosity. Our approach allows for the creation of architected topologies with feature sizes from a few micrometers to millimeters, allowing for a full investigation of the volume size effects. The associated microscale sizedependent mechanical properties of the 3D architected PDC metamaterials are investigated. Compression tests are performed on the as-fabricated metamaterials of 3D octet and cuboctahedron with relative densities ranging from 1 to $22 \%$. The experimental results and analysis indicate that the strength of the parent solid estimated from that of the metamaterials increases as the decrease of relative densities approaching 1\%. This effect came from the decreasing of strut thickness while keeping the strut length constant. We then expanded the investigation of the size-dependent strength as a function of free unit volume of single strut members. This size-dependent strength of the parent solid is discussed using Weibull analysis. It is envisioned that the utilization of the sizedependent mechanical property enabled by the highresolution AM can improve the reliability of this architected structural materials and accelerate the application of the 3D microarchitected high-temperature ceramics for engineering applications.

\section{FABRICATION AND EXPERIMENTAL METHODS}

\section{A. Unit cell structure selection and lattice design}

Due to the different arrangements of cell struts, cellular structures can deform by either bending or stretching of the cell struts and can be classified into bendingdominated or stretch-dominated structures, respectively. Maxwell $^{27}$ proposed an algebraic rule setting out the condition for a pin-jointed frame of $b$ struts and $j$ 

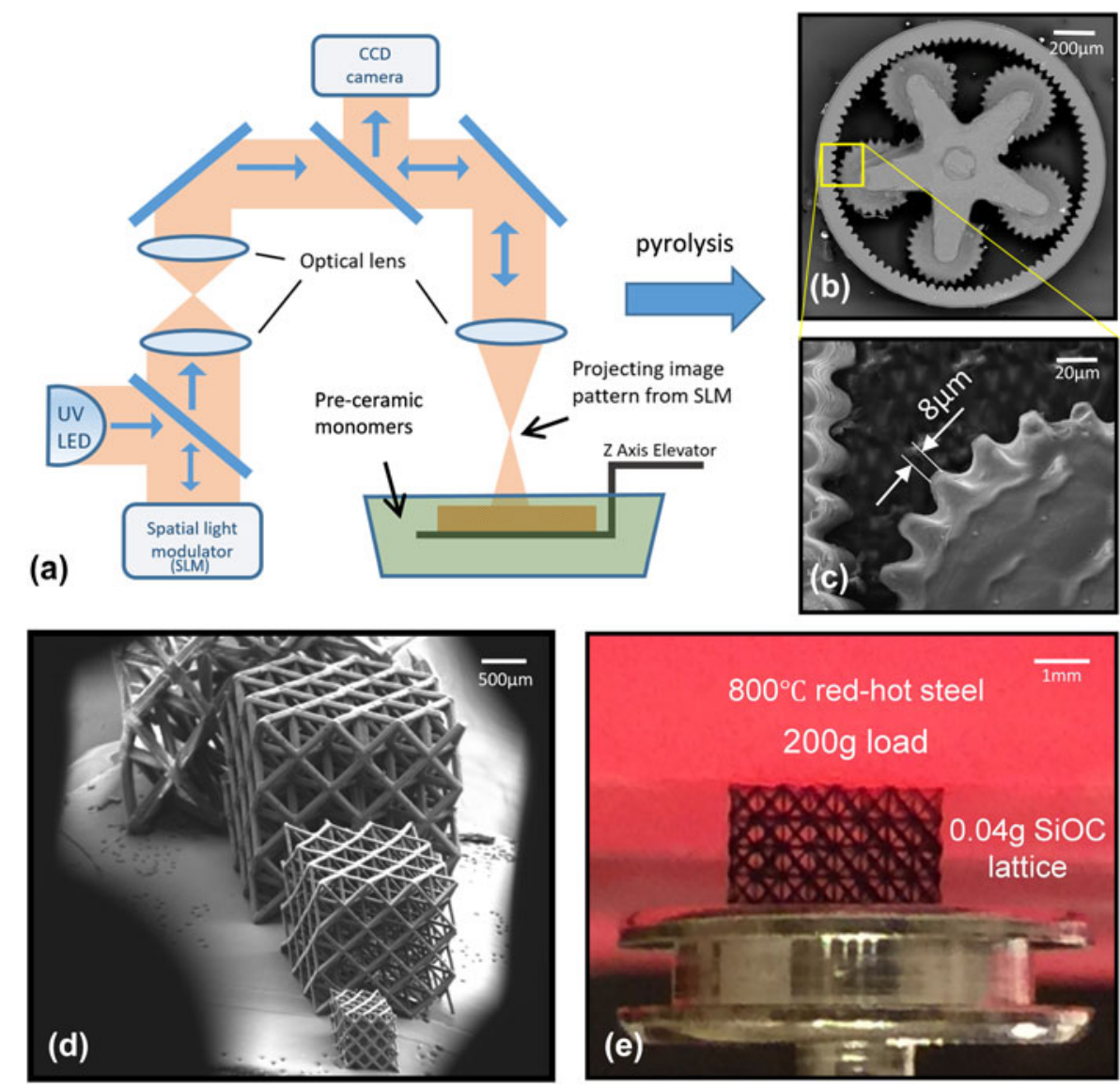

FIG. 1. (a) Schematics of the large-area, high-resolution optical AM system. (b and c) Scanning electron micrographs with different magnifications for the planet gear set made of PDC. (d) Scanning electron micrographs for the lattices with four different sizes. (e) Demonstration of the hightemperature stability of a PDC lattice.

frictionless joints to be both statically and kinematically determinate. In three dimensions, the condition is

$$
M=b-3 j+6=0 .
$$

If its joints are locked (rigid joint) and $M>0$, its members carry tension or compression when loaded, and it becomes a stretch-dominated structure. A stretchdominated unit cell structure is substantially more mechanically efficient than its bending-dominated $(M<$ 0 ) counterpart because the slender structure is much stiffer when stretched than when bent. Thus, we choose stretch-dominated structures to fabricate architected PDC metamaterials.

The octet-truss unit cell, a stretch-dominated structure first proposed by Fuller, ${ }^{28}$ is used in this study. The octettruss structure is a method for filling 3D space with a structurally efficient truss structure of arbitrary cell size [Fig. 2(a)]. The cell has a regular octahedron as its core, surrounded by eight regular tetrahedra distributed on its faces. All the strut elements have identical aspect ratios, with 12 solid rods connected at each node. The cubic symmetry of the cell's $f_{\mathrm{cc}}$ structure generates a material with nearly isotropic behavior. When made from high specific modulus and strength materials, the octet-truss lattice is therefore a weight efficient, stress supporting cellular topology. The cuboctahedron unit cell, composed of a periodic arrangement of octahedra, is also used in this study [Fig. 2(b)]. While it is not fully rigid, the octahedral sub-units are rigid. It is therefore defined to be a periodically rigid topology. ${ }^{29}$

The struts of the unit cells are designed to have a square cross-section, thickness, $t$, and node-to-node length, $l$. The unit cell size equals $\sqrt{2} l$ and the cubic lattices, consisting of $3 \times 3 \times 3$ unit cells, are constructed by periodic packing of these two kinds of unit cells along their three principal directions. Here, we fabricated samples with relative densities ranging from 1 to $22 \%$. The relative densities were tuned by modifying the aspect ratio $(t / l)$ within the periodic unit cells. More details about the relative density calculation are illustrated in Sec. II.B. Among all these lattices, the length, $l$, of each strut and hence the overall dimension of the samples have been kept constant. In addition, octet-truss lattices with the same relative density (same aspect ratio, $t / l$ ) but varying 


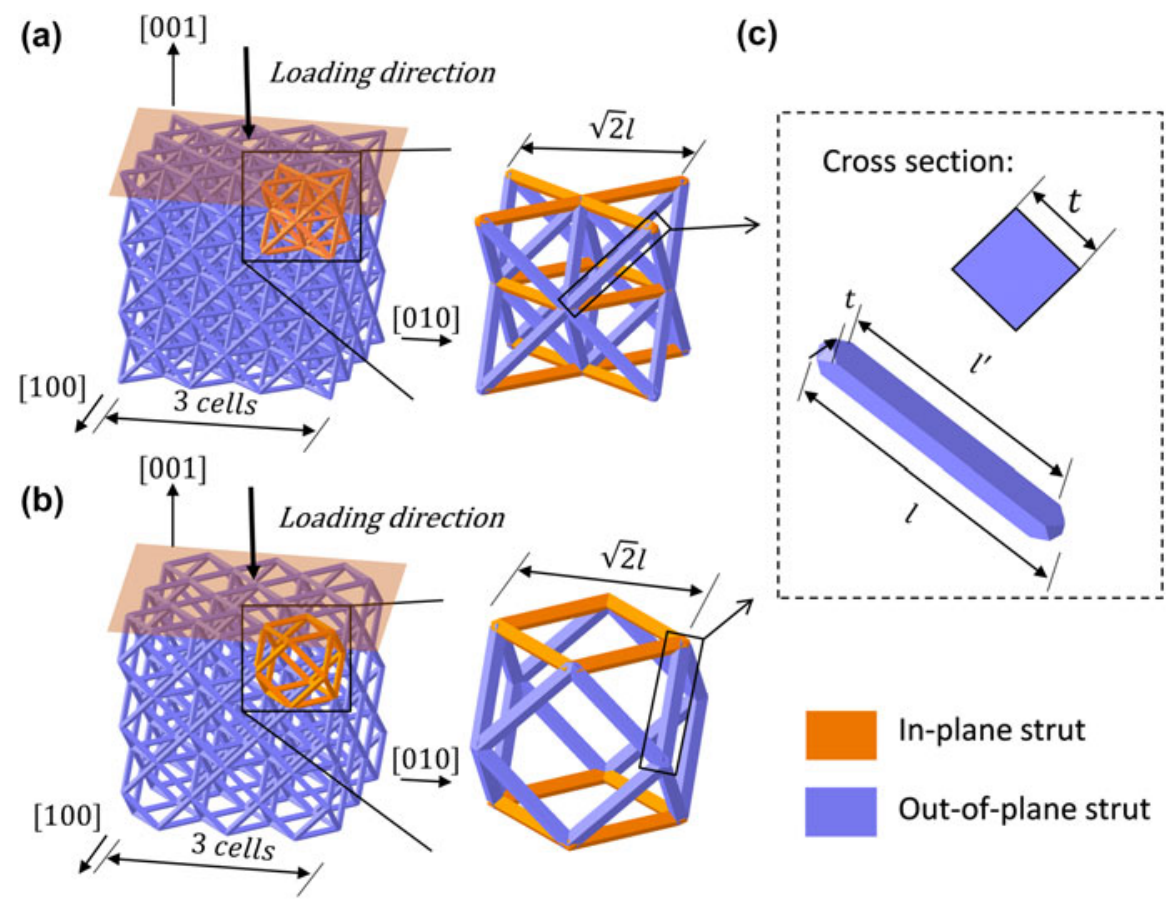

FIG. 2. Computer-aided design models for (a) the octet-truss lattice, unit cell, (b) the cuboctahedron lattice, unit cell, and (c) their struts.

cell size, i.e., strut thickness and strut length, are also fabricated and tested.

\section{B. Relative density design}

The analytical expressions for the relative density of octet-truss and cuboctahedron lattices are derived. For low relative density, the higher order term of the strut aspect ratio, i.e., $t / l$, due to the nodal effect, is negligible; as the strut aspect ratio increases, the nodal effect plays a more significant role in relative density calculation and neglecting this effect renders noticeable overestimation of the relative density. For octet-truss and cuboctahedron lattice with square cross-sections, the node is considered as a cube and hence the volume of the node is taken as $V_{\text {node }}=t^{3}$. The end-to-end length [distance between the edges of two nearest node as shown in Fig. 2(c)] is defined by $l^{\prime}=l-t$, where $l$ is typically called the nodeto-node strut length. By considering a repeating unit cell, the relative density accounting for the nodal effect is then expressed as

$$
\bar{\rho}=\frac{\rho}{\rho_{\mathrm{s}}}=\frac{N_{\text {strut }} t^{2} l^{\prime}+N_{\text {node }} t^{3}}{L^{3}},
$$

where $N_{\text {strut }}$ and $N_{\text {node }}$ are the number of struts and nodes within a repeating unit cell and $L$ is the edge length of a repeating cubic unit cell.

Considering the cases of octet-truss and cuboctahedron unit cells, $N_{\text {strut }}$ is 24 and 12 for octet-truss and cuboctahedron unit cells, respectively; $N_{\text {node }}$ is 4 and 3 for octet-truss and cuboctahedron unit cells, respectively; $L$ is equal to $\sqrt{2} l$. Therefore, the relative density expressions are given by

$$
\begin{aligned}
& \bar{\rho}_{\text {octet }}=6 \sqrt{2} \frac{t^{2}}{l^{2}}\left(1-\frac{5 t}{6} \frac{l}{l}\right), \\
& \bar{\rho}_{\text {cubo }}=3 \sqrt{2} \frac{t^{2}}{l^{2}}\left(1-\frac{3 t}{4} \frac{t}{l}\right),
\end{aligned}
$$

where $\bar{\rho}_{\text {octet }}$ and $\bar{\rho}_{\text {cubo }}$ are the relative densities of octettruss and cuboctahedron lattices with nodal correction, respectively. The corresponding expressions without nodal correction simply neglects the term in the parenthesis in Eqs. (3) and (4). The discrepancy between relative densities with and without nodal correction starts to emerge when $\bar{\rho}>20 \%{ }^{30}$ In our case, we neglect the nodal volume effect since the relative density range used for experiments is $1-22 \%$.

\section{Fabrication of 3D microarchitected PDCs with microsized ligaments}

While the previous work has demonstrated the possibility of printing PDCs, the instability and volatile nature make it challenging for further reduction of feature sizes below 100 micrometers. Here, both vinyl- and thiolsiloxanes were chosen due to their high vinyl and thiol content, respectively. This increases the crosslink density of the system, which simultaneously increases the printed 
preceramic structure stiffness, critical for low-density fabrication, while also eliminating excessive burn-off and shrinkage during pyrolysis to ceramics. Previous PDC studies have utilized thiol-ene chemistries, but either did not report their monomers or utilizes unstable alkoxy silane precursors, which limits shelf life stability. ${ }^{25,31}$ The monomers were combined with a photoinitiator and photo-absorber for high-resolution printing. UV curing occurred through the free radical thiol-ene click reaction. A radical quencher was also added to the preceramic monomer to maintain resin stability before printing. ${ }^{32,33}$ Thiol-ene click chemistry is a highly efficient chemistry, and mixing of monomers without this stabilizer or other stabilizers can readily lead to premature "dark" crosslinking within light-blocked containers, even without the photoinitiator. This dark polymerization has been reported to be likely caused by peroxide impurities and also an ambient oxygen ground state charge transfer process; hence, stabilizers are nearly always required.

A high-resolution, large-area stereolithography system was used for the "green part" (preceramic parts before pyrolysis) fabrication, as shown in Fig. 1(a). The 3D models were first built using a custom code and then sliced into 2D patterns. These 2D patterns are sequentially transmitted to a spatial light modulator, which is illuminated with UV light from a light emitting diode array. Each image is projected through a reduction lens onto the surface of the photosensitive resin. The exposed liquid cures, forming a layer in the shape of the 2D image, and the substrate on which it rests is lowered to reflow a thin film of liquid over the cured layer. The image projection is then repeated, with the next image slice forming the subsequent layer. To increase the resolution, the layer thickness during the $3 \mathrm{D}$ printing process must be well controlled. Experiments were conducted to study the effect of polymerization depth against light power projected onto the liquid surface, demonstrating the reliable printing layer thickness control below 10 micrometers.

To further expand the scalability of the architected metamaterial and examine the size effects under a wide range of structural feature size control, the spatial light modulator is coordinated with an optical scanning system to produce large-scale parts with microscale resolution. As the mirrors scan, 2D patterns are reflected onto a new area next to the previously exposed area. The pattern change on the projector is coordinated with the scanning rate of the scanning mirror system. A customized focusing lens is used below the scanning optics to project the image onto the liquid surface. The frame is updated as the image is moved via the scanning optics to effectively create a continuous image in the photosensitive material. As this scanned image is much larger than a single image of the projector, it enables small feature sizes over a large area. This technique allows the fabrication of preceramic parts, hundreds of millimeters in size, with multiscale 3D architected features down to the $10-\mu \mathrm{m}$ scale [Figs. 1(b) and 1(c)], which is uniquely suitable for fabricating lattice materials with a broad range of feature sizes.

The green parts are then pyrolyzed to form the PDC lattices (Fig. 3). Pyrolysis was carried out in a tube furnace based on previous reports, ${ }^{25}$ ramping at $1{ }^{\circ} \mathrm{C} /$ min, holding at $1000{ }^{\circ} \mathrm{C}$ for $1 \mathrm{~h}$, and then ramping to room temperature at $3{ }^{\circ} \mathrm{C} / \mathrm{min}$ under ultra-high purity argon. Siloxane conversion is accompanied by water, methane, and low-level alkene degasification leaving behind a blend of silica $\left(\mathrm{SiO}_{2}\right)$, carbon, and SiOC depending on the resin carbon content and processing. ${ }^{25}$

\section{Experimental design}

The mass and volume of all the ceramic lattices are measured after pyrolysis. The relative density is calculated as the ratio between the mass and volume and compared with the designed volume fraction. The asfabricated SiOC lattices were tested at ambient temperature in free compression along [001] direction, as shown in Fig. 2, at a nominal strain rate of $8 \times 10^{-4} \mathrm{~s}^{-1}$ on Instron 5944 using standard flat compression plates (T1223-1022 Instron, Norwood, Massachusetts). The peak load divided by the cross-sectional area of the lattices was defined as the effective strength of the lattices. The Young's modulus was extracted from the steady slope of the stress strain curves. The tested strength, Young's modulus of lattices, and relative density of the lattices are used to plot the scaling relationship and size effect analysis in Sec. III.

\section{RESULTS AND ANALYSIS}

\section{A. Polymerization depth characterization}

Traditional liquid resin compositions used for stereolithography usually consist of liquid monomer, photoinitiator, and passively absorbing dye. The photoinitiator will release radicals when exposed to UV light. Solidification of the liquid monomer occurs as a result of cross-linking when it reacts with radicals within the preceramic monomers. Passively absorbing dye such as Sudan is used for absorbing the UV light to well control the curing depth of the liquid monomer. We characterized the working curves of the preceramic monomer through optimization of the photo-absorber and vinylthiol siloxane as well as the addition of the stabilization quencher. The working curve equation ${ }^{34}$ is used to describe the curing depth of the preceramic resin, $C_{\mathrm{d}}$, in a form of 


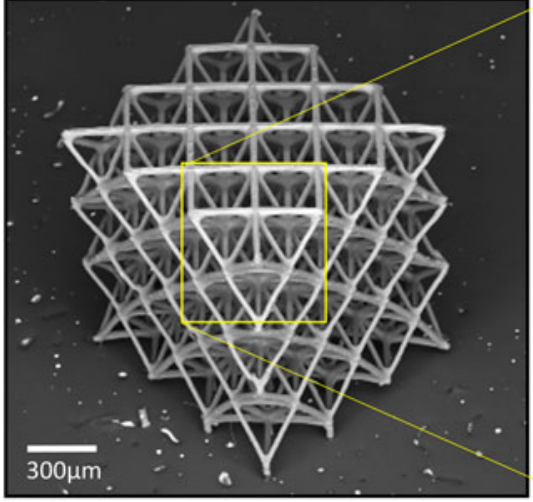

(a)

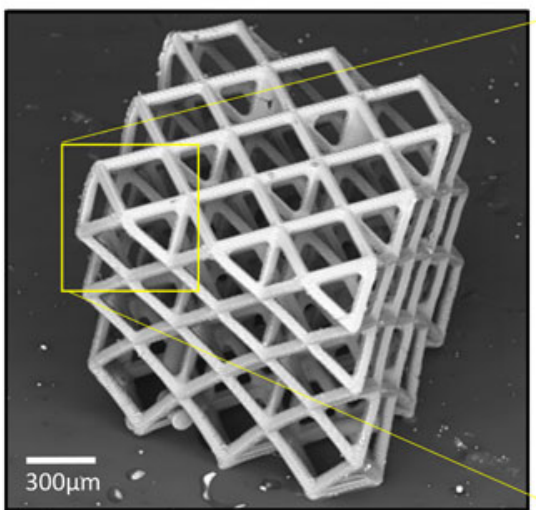

(b)

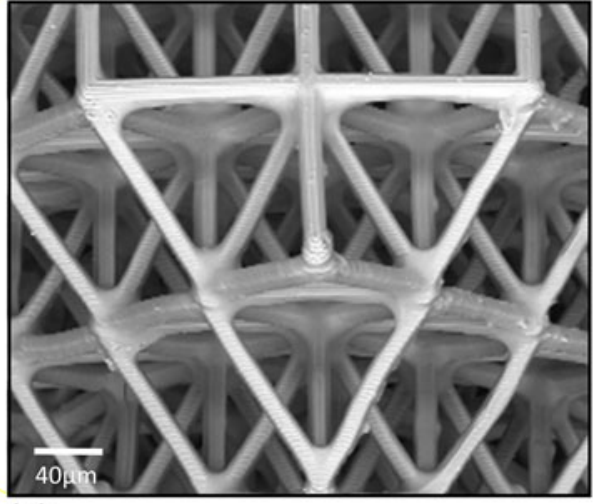

(c)

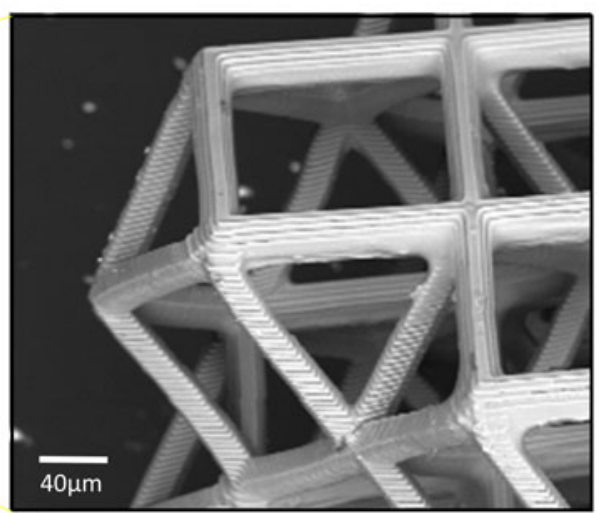

(d)

FIG. 3. ( $a$ and b) Scanning electron micrographs of the octet-truss lattice and the cuboctahedron lattice after pyrolysis. (c and d) Scanning electron micrographs for the unit cell of the octet-truss lattice and the cuboctahedron lattice.

$$
C_{\mathrm{d}}=\frac{1}{\alpha} \ln \left(\frac{\alpha_{\mathrm{I}} E}{E_{\mathrm{c}}}\right)
$$

where $\alpha=\alpha_{\mathrm{I}}+\alpha_{\mathrm{D}}$ is the resin absorption coefficient, $\alpha_{\mathrm{I}}$ is the absorption coefficient of the photoinitiator, $\alpha_{D}$ is the coefficient of the passively absorbing dye, $E$ is the actual incident energies, and $E_{\mathrm{c}}$ is the critical incident energy needed for cure, in units of $\mathrm{mJ} / \mathrm{cm}^{2}$.

To characterize the polymerization depth of a free layer, an array of overhanging bridge structures was fabricated with an array of photon energy [Fig. 4(a), inset]. Each bridge layer represented one polymerization layer, and the mean thickness of a given layer was taken to be the polymerization depth. Figure 4(a) shows the variation of the polymerization depth as a function of total exposure energy $\left(\mathrm{mJ} / \mathrm{cm}^{2}\right)$ received by the liquid resin. The results indicate that the polymerization depth is linearly proportional to the natural logarithm of UV exposure energy, which is in good agreement with the numerical model. The experimental results confirmed the needed exposure energy for reducing the polymerization depth, which are needed for reliably creating final feature size below $10 \mu \mathrm{m}$.

\section{B. Polymer-derived ceramic characterization}

The pyrolysis procedure was accompanied by $37 \%$ weight loss and $34 \%$ linear shrinkage. Scanning electron microscopy with energy dispersive X-ray analysis (EDS) was performed. The result [Fig. 4(b)] shows that the sample has a composition of 20.44 atomic percent (at.\%) carbon, 38.6 at.\% oxygen, and 40.96 at.\% silicon, or $\mathrm{SiO}_{0.9} \mathrm{C}_{0.5}$ The content of oxygen is similar to the previous studies; however, the content of carbon is lower and the content of silicon is higher compared to the previous studies. $^{25}$ Previous reports have shown the significant dependence of SiOC on composition. ${ }^{35}$ These effects can be generalized as the $\mathrm{SiOC}$ becomes closer in composition to silicon carbide ( $\mathrm{SiC}$ ), i.e the elimination of oxygen, the greater the mechanical properties, Young's modulus and hardness. The mechanical properties of $\mathrm{SiC}$ are approximately double that of SiOC. Variation in composition can lead to an approximate doubling in properties.

\section{Strength and Young's modulus scaling in high- temperature ceramic lattices}

The testing results of Young's modulus and strength of both octet-truss and cuboctahedron are summarized in 

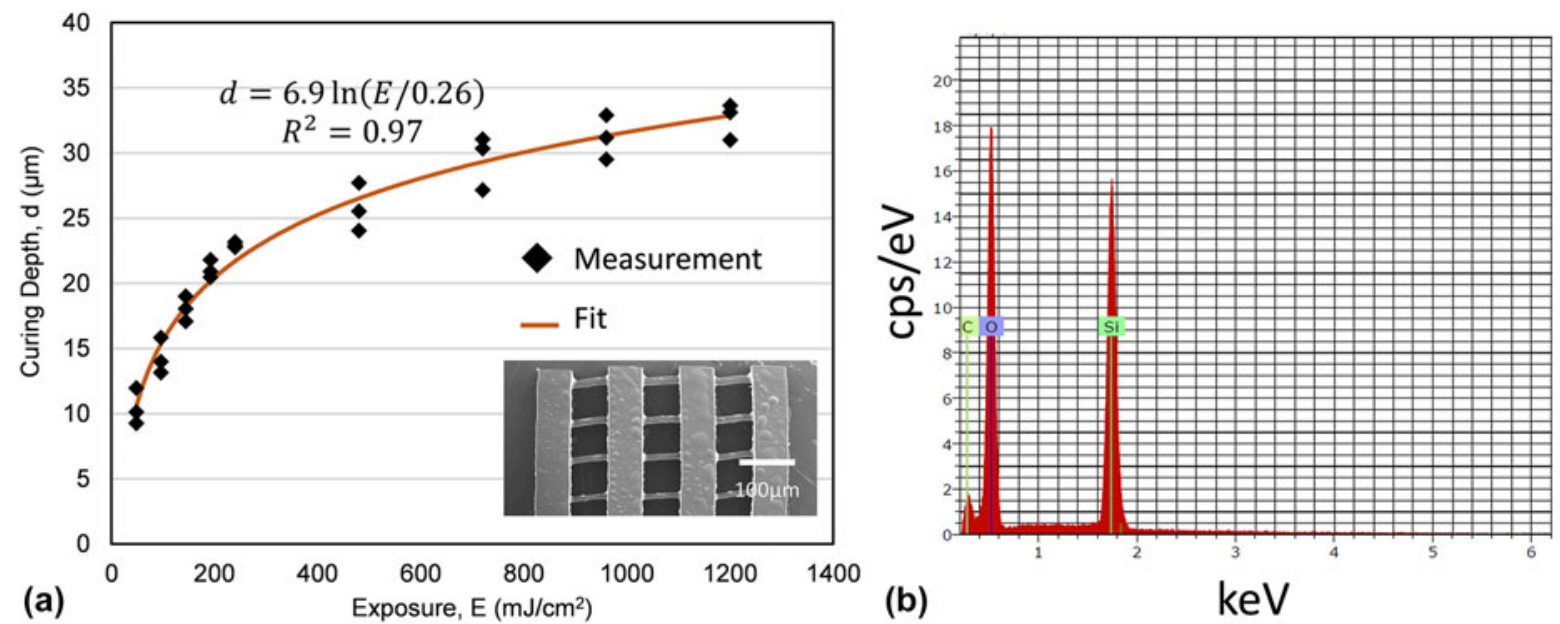

FIG. 4. (a) Photo flux versus polymerization depth. The curing depth increases linearly with natural logarithm of exposure energy. (b) EDS of a single strut inside the lattice.
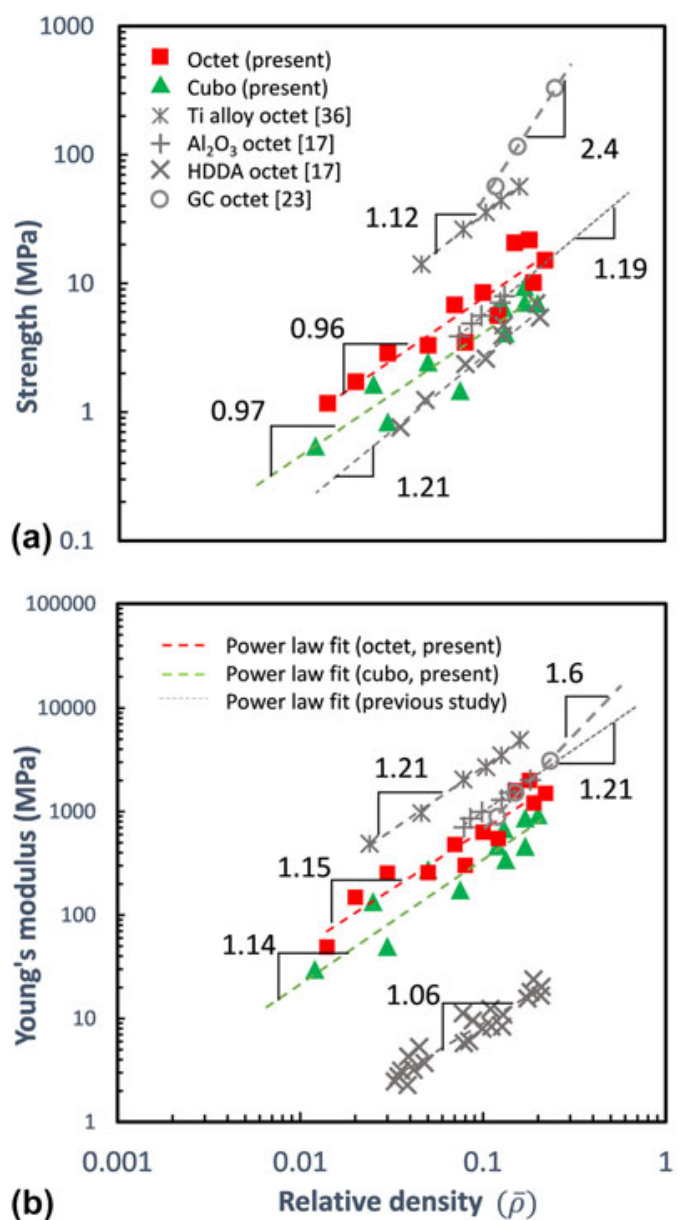

FIG. 5. Scaling law of effective strength and Young's modulus of the octet-truss and cuboctahedron lattices. The experimental results of previous studies are shown as a comparison.

Fig. 5. The compressive strength versus relative density is plotted for each architecture in Fig. 5(a) and compared to other octet-truss lattices with different parent solids. ${ }^{17,36}$
Well-developed theories ${ }^{36,37}$ show that on the macroscale, under uniaxial compressive loading, the compressive stiffness and yield strength of these structures theoretically show linear scaling relationships: $E / E_{\mathrm{S}} \propto \bar{\rho}$ and $\sigma / \sigma_{\mathrm{s}} \propto \bar{\rho}$, where $E_{\mathrm{s}}$ is the Young's modulus of the base material and $\sigma_{\mathrm{s}}$ is the strength of the base material. However, as indicated in Fig. 5(a), the previously reported experimental results ${ }^{17,36}$ show that under uniaxial compression, the scaling factors for stretch-dominated lattices are normally in the range of 1.06-1.21, or even as high as 2.4 , larger than the theoretical value, since there are fabrication imperfections weakening the performance of these lattices.

Interestingly, in testing results of the PDC lattices, the apparent strength $(\sigma)$-relative density $(\bar{\rho})$ scaling in the as-fabricated ceramic lattices shows a scaling power that is smaller than 1 for both octet-truss lattices and cuboctahedron lattices, outperforming theoretically predicted scaling powers. By contrast, the obtained scaling relationship of Young's modulus $(E)$ and relative density $(\bar{\rho})$ in Fig. 5(b) is consistent with previously reported scaling values as well as predicted by theory on stretchdominated lattices. It should be commented that the cuboctahedron lattice here is considered to be a stretchdominated lattice for its periodic rigidity. We hypothesize that the strength of these architected lattices are influenced by possible strong size-dependent effects as the smallest ligament becomes slender in the microscale range (i.e., the reduction of strut size), which will be further investigated in the following sections.

\section{Strut thickness-dependent PDC strength}

To investigate the size dependency of PDC strength, the strength of the parent solid that comprises the individual solid strut members for each sample is estimated by normalization of the effective compressive 
strength of the lattices with its topology-dependent factor. The effective strength of cellular materials can be approximated by the first-order scaling law ${ }^{38}$ :

$$
\sigma_{\text {eff }}=\Sigma \sigma_{\mathrm{s}},
$$

where $\Sigma$ is a lattice topology and geometry dependent scaling factor, and $\sigma_{\mathrm{s}}$ is the fracture strength of the base material. The scaling factor $\Sigma$, accounting for both the lattice geometry and relative density, can be calculated by

$$
\Sigma=C \bar{\rho}^{\alpha}
$$

where $C$ is a geometric parameter depending on the lattice topology as well as the loading direction, $\bar{\rho}$ is a function of $(t / l)$, and the exponent $\alpha$ is 1 for stretchdominated behavior, theoretically. ${ }^{39}$ Based on previous studies, the experimental results of the geometric parameter, $C$, for octet-truss lattices and cuboctahedron lattices are 0.3 and $0.16^{29,37}$ for [001] direction loading, respectively.

Such that the effective strength of the lattice can be normalized by the geometric factor to estimate the strength of the individual ligament

$$
\sigma_{\mathrm{n}}=\frac{\sigma_{\text {eff }}}{\Sigma},
$$

where $\sigma_{\mathrm{n}}$ can be seen as an estimation of the strength of the parent solid.

Here, as relative density reduces, the only changing parameter for both octet-truss lattices and cuboctahedron lattices is strut thickness while the strut length has been kept constant. The normalized strength of the parent solid excluding all geometric factors is plotted against the only variable strut thickness in Fig. 6. The size-dependent mechanical property of lattices with differing relative density from Fig. 5(a) can now be visualized in Fig. 6 . The means of estimated PDC strength are calculated using each $10-\mu \mathrm{m}$ interval for strut thickness for strut volume to filter the scattering of the data. ${ }^{40}$ The strength of the PDC solid carries a power-law relationship with the strut thickness as there is no characteristic length for the brittle PDC strut. ${ }^{40-43}$ A power-law fitting is applied to the calculated mean strength of the ceramic solids as a function of strut thickness and yields the following relationship:

$$
\sigma_{\mathrm{s}}=D t^{-\beta},
$$

where $\beta$ is the scaling factor of the strut thickness and is found to be 0.36 , from our measured experimental results, while $D$ is a constant. The normalized PDC strength increases as the strut thickness decreases, as shown in Fig. 6, indicating a size dependency of the strength of the PDC solids possibly due to the reduced size and number of cracks.

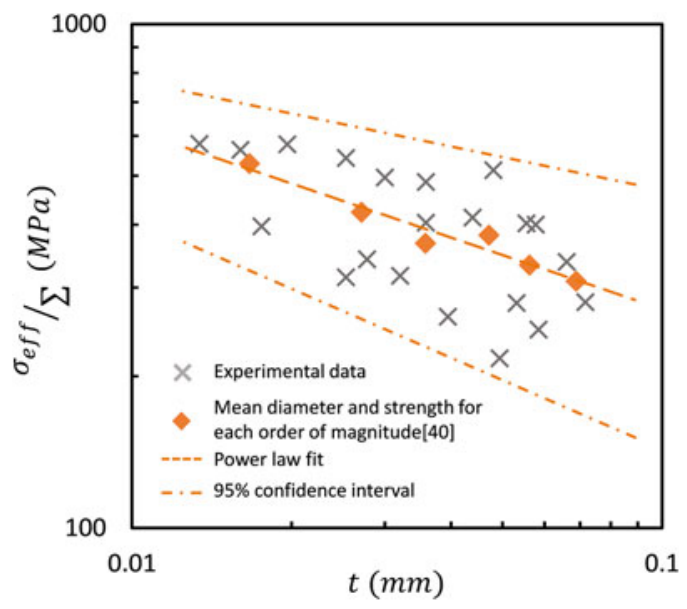

FIG. 6. Effect of strut diameter on the normalized strength of relativedensity-controlled ceramic lattices. The means of estimated PDC strength are calculated using each $10-\mu \mathrm{m}$ interval for strut thickness for strut volume to filter the scattering of the data.

Previous studies also show that the strength of brittle ${ }^{44-45}$ materials typically increases with decreasing dimensions. Based on the relationship proposed by Griffith between the fracture strength, $\sigma_{f}$, and the critical size of a flaw, $c$, for brittle materials such as ceramics, ${ }^{46}$ we know that

$$
\sigma_{\mathrm{f}} \propto \frac{1}{\sqrt{c}}
$$

A flaw cannot be larger than the component in which it is located. Assuming $c$ correlates with the strut thickness, $t,{ }^{47}$ the relationship can be written as

$$
\sigma_{\mathrm{f}} \propto t^{-0.5} .
$$

Even though the scaling factor from our measured experimental results is lower than the value proposed by Griffith, which may be caused by the insufficient number of measurements and experimental errors, our experimental results indicate a size dependency of the strength of the PDC solids.

This size effect as a function of reduced strut thickness in density-controlled sample explains the apparent scaling law of $\sigma_{\text {eff }}-\bar{\rho}$. By combining Eqs. (6), (7), and (9), the effective strength of the lattice can then be modified as

$$
\sigma_{\text {eff }}=C D \bar{\rho}^{\alpha} t^{-\beta}
$$

Substitute relative density expression without nodal correction into Eq. (12), we get

$$
\sigma_{\mathrm{eff}}=C D \bar{\rho}^{\alpha-\frac{\beta}{2}} l^{-\beta} .
$$

Equation (13) can be further modified as 


$$
\sigma_{\text {eff }} \sim \bar{\rho}^{\alpha-\frac{\beta}{2}}
$$

From Eq. (14), we know that the scaling between the effective strength and relative density of the lattice is reduced by a factor of $-\frac{\beta}{2}$, which is caused by the size dependency of the PDC strength. As density further reduces, this $\frac{\beta}{2}$ will further increases, which will result in higher specific strength in lower density solid architected ceramics as compared to higher density samples, i.e., an apparent scaling power smaller than 1 as density decreases further.

\section{E. Strut volume-dependent PDC strength analogous to Weibull size effect}

We then proceed to investigate the size strengthening effect by measuring the strength of lattices of identical relative densities but with uniform reduction of minimal strut volumes. While the benefit of reducing the coating thickness of ceramics through ALD has been experimentally demonstrated in previous studies in Refs. 48 and 49 and explained by the weakest link theory, ${ }^{50}$ the role of the overall size, i.e., strut volume of each solid ligament that comprise the overall lattices, is not clear. The size from the length-direction, which contributes to the surface volume of individual solid strut members, in addition to the diameter and thickness of the strut could contribute the overall size effect of the lattice material. Here, to fully capture the size dependency of the PDC strength, we expand the study by simultaneously modifying the average strut thickness and length within the unit cells. The samples were fabricated with identical relative densities but with a serial reduction of single strut volumes. The Weibull statistical analysis is applied to interpret the relationship between strut volume and material strength.

Derived from the weakest link theory based on a chain model ${ }^{41,42}$ the risk of failure of the material is given by a probabilistic expression:

$$
P_{\mathrm{f}}(\sigma)=1-\exp \left\{-\frac{P(\sigma) V}{V_{0}}\right\},
$$

where $P_{\mathrm{f}}(\sigma)$ represents the risk of failure of a material under stress $\sigma ; V$ is the volume of the material and $V_{0}$ is a standardized reference volume; $P(\sigma)$ is defined as the probability of material failure under stress $\sigma$ for a given material volume $V$. Weibull ${ }^{51,52}$ proposed a power-law solution for $P(\sigma)$, vanishing at a critical value of $\sigma_{\mathrm{c}}$ as $P(\sigma)=\left(\frac{\sigma-\sigma_{\mathrm{c}}}{\sigma_{0}}\right)^{m}$, where $\sigma_{\mathrm{c}}$ is the critical strength below which the probability of failure is zero, for brittle material $\sigma_{\mathrm{c}}=0$ since for brittle materials, any tensile stress can cause brittle fracture; $\sigma_{0}$ is the characteristic strength at $P_{\mathrm{f}}$ $(\sigma)=63.2 \% ; m$ is the well-known Weibull modulus. ${ }^{53}$
Therefore,

$$
P_{\mathrm{f}}(\sigma)=1-\exp \left\{-\left(\frac{\sigma}{\sigma_{0}}\right)^{m} \frac{V}{V_{0}}\right\} .
$$

For the same risk of failure under two different stresses, we obtain $\frac{\sigma_{1}}{\sigma_{2}}=\left(\frac{V_{2}}{V_{1}}\right)^{\frac{1}{m}}$, which leads to the expression of nominal strength of material,

$$
\bar{\sigma}_{\mathrm{f}} \propto\left(\frac{1}{V}\right)^{\frac{1}{m}} .
$$

In the present case, $V=t^{2} l$ represents the strut volume. Volume is a function of strut thickness $(t)$ as defined in Fig. 2.

According to the theorem as in Eq. (17), the strength of PDC relates to the strut volume by a simple power law from a statistical viewpoint as shown in Fig. 7, which plots the normalized PDC strength versus the strut volume $(V)$. The mean of the estimated strength was calculated using each order of magnitude for the strut volume. The scaling power in Fig. 7 shows the trend of the size dependency of strength as a function of strut volume reduction over three decades: decrease of the strut volume leads to the increase of the PDC strength. For 3D architected brittle ceramic metamaterials, the failure of the metamaterials is determined by successive failure of multiple struts similar to the case in Ref. 58. The failure of individual strut is catastrophic due to the brittle nature of PDC, which suggests the applicability of the Weibull analysis and weakest link theory only on the strut volume instead of the total volume of the architected material. ${ }^{52}$ However, to obtain the Weibull modulus and size effect for 3D microarchitected brittle ceramic metamaterials, a nonclassical

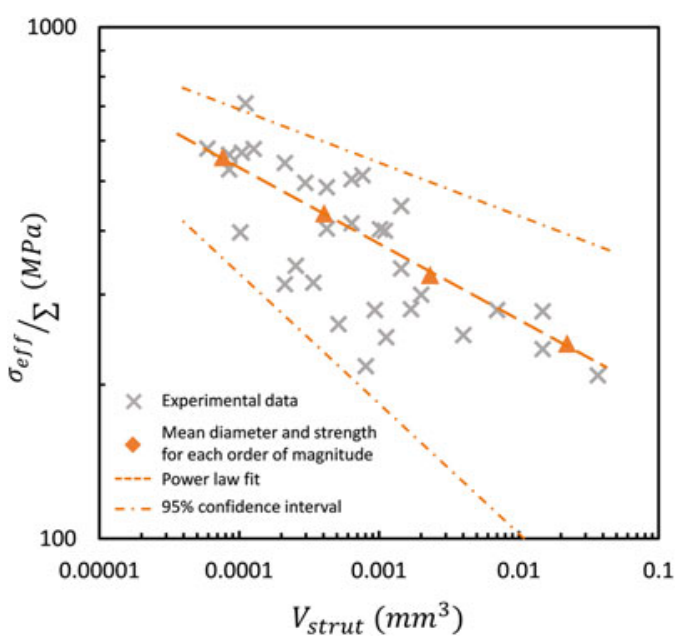

FIG. 7. Effect of strut volume on the normalized strength of volumecontrolled ceramic lattices. The mean of the estimated strength was calculated using each order of magnitude for the strut volume. 
probabilistic model is needed to account for structural factors, which is discussed in Sec. IV.

\section{DISCUSSION}

This work reports the fabrication of high-temperature ceramic-architected metamaterials and the characterizations of the size effects derived from the solid microscale struts that comprise the stretch-dominated lattices. AM of polymer-derived ceramics had been recently reported to process virtually no porosities in as-fabricated ceramic components. This work, for the first time, extends this paradigm to the fabrication of high-temperature precision ceramics with solid microscale features, opening up new opportunity to capitalize on the size effects from miniaturized features and proliferate them to lightweight ceramic-architected materials. A well-controlled stabilized preceramic light sensitive monomer with UV stabilizers enables a confinement of polymerization depth within 10 micrometers, resulting in reliable production of precision 3D ceramic components comprised of microscale solid features.

While extensive investigations have reported the stiffness and strength for a limited number of test samples $^{37,39,54}$ with thin-walled hollow tube ceramics, this study provides direct observations of the size-dependent strength of ceramic microarchitected metamaterials with solid strut members. The as-fabricated stretch-dominated $\mathrm{SiOC}$ ceramic metamaterials have revealed a strong size dependency in relative densities reduced from 22 to $1 \%$. A breakdown of strength as a function of the diameter of the ligaments revealed a stronger ligament strength when density of the lattice is reduced. This observation was corroborated by architected lattices with varying single strut volumes of identical relative densities. We conclude that these high-temperature metamaterials approximately follow a Weibull distribution of strength as a function of reduced single strut volumes.

While the present work provides insights of single strut volume on the contribution of the strength of architected metamaterials, it did not capture the size effects of architected metamaterials comprised of a very large number of unit cells, i.e., the cell number size effects. Likewise, the weakest link theory that has been used to relate to the size effects of architected metamaterials will not fully describe the scenario of large-area architected metamaterials comprised of a large number of cells. This limitation can be summarized in 2-fold: (i) while the classic Weibull analysis ${ }^{42,52}$ as illustrated in Eq. (16) can give insight into the probabilistic distribution of strength and "the smaller the stronger" size effects on solid ceramic-architected metamaterials, it is not entirely applicable as the structural members with different orientation and position in the architectural layout may carry different stresses such that the effect of stress distribution among strut members should be taken into account. ${ }^{55}$ (ii) As indicated in Sec. V, the Weibull size effect originated from the weakest link theory assumes that the failure of architected metamaterials is dictated by the failure of any single strut member. This assumption holds for microlattices with a limited number of unit cells; it does not necessarily apply to lattices with a significantly larger number of unit cells. The actual strength extracted from the experimental stress-strain curve is determined by the successive or nearly catastrophic failure of multiple struts. In lattices with a large number of unit cells, the successive failure of struts within the metamaterial is dominated by the effective fracture toughness of the lattice materials, which gives the metamaterial resistance to crack initiation with existing flaws (i.e., breaking of a single strut member or a few strut members). ${ }^{54,56}$ The subject of the fracture toughness of architected metamaterials remains as a future topic of investigation.

It is interesting to note that the Weibull size effects also did not fully describe the size effects in recently investigated quasi-brittle ceramic lattice networks attributed to the stress distribution throughout the entire material system, which is analogous to the 3D microarchitected brittle ceramic metamaterials. A number of studies have proposed different models utilizing series coupling of elements $^{57}$ in which a number of chain models are arranged in series, instead of those arranged in parallel, to represent that a single failure of the chain model does not fail the entire system, in addition to local failure events of a ceramic unit cell dictated by the weakest link theory. ${ }^{42,58,59}$ Previous studies show an analytical model to deal with the cellular ceramic successive failure by utilizing the homogenization method on the material system scale to consider the failure probability of small-scale constitutive elements on which the classical Weibull theory is applied. Their proposed framework may be extended to the architected ceramic metamaterials and hence involves representations at both microscale (single strut) and macroscale (lattice), which could be analogous to micro- and nano-architected ceramics containing a large number of cells. Nevertheless, to fully capture the size effect of architected metamaterials studied in this work, future efforts will include incorporating (i) a sufficiently larger sample number $(>30$ at each strut volume) to derive the accurate Weibull modulus of the strength size effect ${ }^{60}$ and (ii) probabilities of failure strength as a function of unit cell numbers within a metamaterial with constant single strut volumes.

\section{CONCLUSION}

We have demonstrated the AM of precision polymerderived ceramic components with complex, microscale solid features within 10 micrometers. The methods are 
based on large-area projection microstereolithography of stabilized UV-sensitive preceramic monomers with precise control of structural features from $10-\mu \mathrm{m}$ scale to millimeters. To study the strength of the high-temperature SiOC-architected metamaterials, lattices with relative densities ranging from as low as 1 to $22 \%$ were fabricated and characterized through uniaxial compression. We found that at lower densities, the strength of these solid ceramic lattices benefits from the reduced strut thickness at the microscale, suggesting a possible strong sizedependent strength of the strut members. Weibull theory is then utilized to map this dependency with varying single strut volumes in the as-fabricated lattice materials with identical relative densities. Our study provides the direct observations and evidence suggesting the benefits of microscale structural members in improving the strength of low-density high-temperature ceramics. Through capitalizing upon the reduction of unit strut volumes within the architecture, high-temperature ceramics could achieve high specific strength with only one fraction of their solid counterparts.

\section{ACKNOWLEDGMENTS}

This research was supported by the NSF CMMI1727492, ICTAS Junior Faculty Award, and Startup support from Virginia Tech. The authors would like to thank Hesham Elmkharram, Carlos Suchicital, and Thomas Staley for their technical assistance on post processing. The precision high temperature ceramic printing has been officially filed for a patent.

\section{REFERENCES}

1. K. Wei, X.M. Cheng, F.H. Mo, W.B. Wen, and D.N. Fang: Design and analysis of integrated thermal protection system based on lightweight $\mathrm{C} / \mathrm{SiC}$ pyramidal lattice core sandwich panel. Mater. Des. 111, 435 (2016).

2. S. Bapanapalli, O. Martinez, C. Gogu, B. Sankar, R. Haftka, and M. Blosser: (Student paper) Analysis and design of corrugatedcore sandwich panels for thermal protection systems of space vehicles. In 47th AIAA/ASME/ASCE/AHS/ASC Structures, Structural Dynamics, and Materials Conference (American Institute of Aeronautics and Astronautics, Newport, Rhode Island, 2006), doi: 10.2514/6.2006-1942.

3. K. We, R.J. He, X.M. Cheng, R.B. Zhang, Y.M. Pei, and D.N. Fang: Fabrication and mechanical properties of lightweight $\mathrm{ZrO}_{2}$ ceramic corrugated core sandwich panels. Mater. Des. 64, 91 (2014).

4. K. Wei, R. He, X. Cheng, R. Zhang, Y. Pei, and D. Fang: A lightweight, high compression strength ultra high temperature ceramic corrugated panel with potential for thermal protection system applications. Mater. Des. 66(Part B), 552 (2015).

5. F. Monteverde and L. Scatteia: Resistance to thermal shock and to oxidation of metal diborides-SiC ceramics for aerospace application. J. Am. Ceram. Soc. 90, 1130 (2007).

6. Y. Matsumura: Stabilization of $\mathrm{Cu} / \mathrm{ZnO} / \mathrm{ZrO}_{2}$ catalyst for methanol steam reforming to hydrogen by coprecipitation on zirconia support. J. Power Sources 238, 109 (2013).
7. N. Tamai, A. Myoui, T. Tomita, T. Nakase, J. Tanaka, T. Ochi, and H. Yoshikawa: Novel hydroxyapatite ceramics with an interconnective porous structure exhibit superior osteoconduction in vivo. J. Biomed. Mater. Res. 59, 110 (2002).

8. M. Peroglio, L. Gremillard, J. Chevalier, L. Chazeau, C. Gauthier, and T. Hamaide: Toughening of bio-ceramics scaffolds by polymer coating. J. Eur. Ceram. Soc. 27, 2679 (2007).

9. R.C. Buchanan: Ceramic Materials for Electronics, 3rd ed. (Taylor \& Francis, New York, New York, 2004).

10. N. Ibanez-Garcia, C.S. Martinez-Cisneros, F. Valdes, and J. Alonso: Green-tape ceramics. New technological approach for integrating electronics and fluidics in microsystems. TrAC, Trends Anal. Chem. 27, 24 (2008).

11. J. Rodel, A.B.N. Kounga, M. Weissenberger-Eibl, D. Koch, A. Bierwisch, W. Rossner, M.J. Hoffmann, R. Danzer, and G. Schneider: Development of a roadmap for advanced ceramics: 2010-2025. J. Eur. Ceram. Soc. 29, 1549 (2009).

12. P. Muralt: Recent progress in materials issues for piezoelectric MEMS. J. Am. Ceram. Soc. 91, 1385 (2008).

13. J-B. Waldner: Nanocomputers and Swarm Intelligence (WileyIEEE Press, New York, New York, 2008).

14. A. Koller: Structure and Properties of Ceramics (Elsevier, Amsterdam, the Netherlands, 1994).

15. B.R. Lawn: Fracture of Brittle Solids, 2nd ed. (Cambridge University Press, 1993).

16. A.G. Evans: Structural reliability-A processing-dependent phenomenon. J. Am. Ceram. Soc. 65, 127 (1982).

17. X.Y. Zheng, H. Lee, T.H. Weisgraber, M. Shusteff, J. DeOtte, E.B. Duoss, J.D. Kuntz, M.M. Biener, Q. Ge, J.A. Jackson, S.O. Kucheyev, N.X. Fang, and C.M. Spadaccini: Ultralight, ultrastiff mechanical metamaterials. Science 344, 1373 (2014)

18. L.R. Meza, S. Das, and J.R. Greer: Strong, lightweight, and recoverable three-dimensional ceramic nanolattices. Science $\mathbf{3 4 5}$, 1322 (2014).

19. X.Y. Zheng, J. DeOtte, M.P. Alonso, G.R. Farquar, T. Weisgraber, S. Gemberling, H. Lee, N. Fang, and C.M. Spadaccini: Design and optimization of a light-emitting diode projection micro-stereolithography three-dimensional manufacturing system. Rev. Sci. Instrum. 83, 125001-1 (2012).

20. X.Y. Zheng, W. Smith, J. Jackson, B. Moran, H.C. Cui, D. Chen, J.C. Ye, N. Fang, N. Rodriguez, T. Weisgraber, and C.M. Spadaccini: Multiscale metallic metamaterials. Nat. Mater. 15, 1100 (2016).

21. J. Bauer, L.R. Meza, T.A. Schaedler, R. Schwaiger, X. Zheng, and L. Valdevit: Nanolattices: An emerging class of mechanical metamaterials. Adv. Mater. 21, 1701850 (2017).

22. L.C. Montemayor, W.H. Wong, Y.W. Zhang, and J.R. Greer: Insensitivity to flaws leads to damage tolerance in brittle architected meta-materials. Sci. Rep. 6, (2016).

23. J. Bauer, A. Schroer, R. Schwaiger, and O. Kraft: Approaching theoretical strength in glassy carbon nanolattices. Nat. Mater. 15 438 (2016).

24. A.J. Jacobsen, S. Mahoney, W.B. Carter, and S. Nutt: Vitreous carbon micro-lattice structures. Carbon 49, 1025 (2011).

25. Z.C. Eckel, C.Y. Zhou, J.H. Martin, A.J. Jacobsen, W.B. Carter, and T.A. Schaedler: 3D printing: Additive manufacturing of polymer-derived ceramics. Science 351, 58 (2016).

26. J.M. Hundley, Z.C. Eckel, E. Schueller, K. Cante, S.M. Biesboer, B.D. Yahata, and T.A. Schaedler: Geometric characterization of additively manufactured polymer derived ceramics. Addit. Manuf. 18(Suppl. C), 95 (2017).

27. J.C. Maxwell: On the calculation of the equilibrium and stiffness of frames. Philos. Mag. 27, 294 (1864).

28. R.B. Fuller: Octet truss. U.S. Patent No. 2986241 (1961). 
29. L.R. Meza, G.P. Phlipot, C.M. Portela, A. Maggi, L.C. Montemayor, A. Comella, D.M. Kochmann, and J.R. Greer: Reexamining the mechanical property space of threedimensional lattice architectures. Acta Mater. 140(Suppl. C), 424 (2017).

30. L.J. Gibson and M.F. Ashby: Cellular Solids: Structure and Properties (Cambridge University Press, Cambridge, U.K., 1999).

31. E. Zanchetta, M. Cattaldo, G. Franchin, M. Schwentenwein, J. Homa, G. Brusatin, and P. Colombo: Stereolithography of SiOC ceramic microcomponents. Adv. Mater. 28, 370 (2016).

32. M.A. Rakas and A.F. Jacobine: Mechanical and dynamic mechanical-properties of photocrosslinked norbornene-thiol copolymer films. J. Adhes. 36, 247 (1992).

33. A. Lowe and C. Bowman: Thiol-x chemistries in polymer and materials science. RSC Polym. Chem. Ser. 6, 1 (2013).

34. J.R. Tumbleston, D. Shirvanyants, N. Ermoshkin, R. Janusziewicz, A.R. Johnson, D. Kelly, K. Chen, R. Pinschmidt, J.P. Rolland, A. Ermoshkin, E.T. Samulski, and J.M. DeSimone: Continuous liquid interface production of 3D objects. Science 347, 1349 (2015).

35. P. Du, X.N. Wang, I.K. Lin, and X. Zhang: Effects of composition and thermal annealing on the mechanical properties of silicon oxycarbide films. Sens. Actuators, A 176, 90 (2012).

36. L. Dong, V. Deshpande, and H. Wadley: Mechanical response of Ti-6Al-4V octet-truss lattice structures. Int. J. Solids Struct. 6061, 107 (2015).

37. V.S. Deshpande, N.A. Fleck, and M.F. Ashby: Effective properties of the octet-truss lattice material. J. Mech. Phys. Solids 49, 1747 (2001).

38. P. Mocingkharnklang, D.M. Elzey, and H.N.G. Wadley: Titanium matrix composite lattice structures. Composites, Part A 39, 176 (2008).

39. V.S. Deshpande, M.F. Ashby, and N.A. Fleck: Foam topology bending versus stretching dominated architectures. Acta Mater. 49, 1035 (2001).

40. O. Flores, R.K. Bordia, S. Bernard, T. Uhlemann, W. Krenkel, and G. Motz: Processing and characterization of large diameter ceramic SiCN monofilaments from commercial oligosilazanes. RSC Adv. 5, 107001 (2015).

41. Z.P. Bazant: Scaling laws in mechanics of failure. J Eng MechAsce. 119, 1828 (1993).

42. Z.P. Bazant: Size effect on structural strength: A review. Arch Appl Mech. 69, 703 (1999).

43. T.C. Tsu, R.A. Mugele, and F.A. Mcclintock: A statistical distribution function of wide applicability. J Appl Mech-T Asme. 19, 233 (1952).

44. F. Riley: Structural Ceramics: Fundamentals and Case Studies (Cambridge University Press, Cambridge, U.K., 2009).
45. P. Chantikul, S.J. Bennison, and B.R. Lawn: Role of grain-size in the strength and R-curve properties of alumina. J. Am. Ceram. Soc. 73, 2419 (1990).

46. A.A. Griffith: The phenomena of rupture and flow in solids. Philos. Trans. R. Soc. London, Ser. A 221, 163 (1921).

47. H.J. Gao, B.H. Ji, I.L. Jager, E. Arzt, and P. Fratzl: Materials become insensitive to flaws at nanoscale: Lessons from nature. Proc. Natl. Acad. Sci. U. S. A. 100, 5597 (2003).

48. R. Liontas and J.R. Greer: 3D nano-architected metallic glass: Size effect suppresses catastrophic failure. Acta Mater. 133, 393 (2017).

49. L.R. Meza, A.J. Zelhofer, N. Clarke, A.J. Mateos, D.M. Kochmann, and J.R. Greer: Resilient 3D hierarchical architected metamaterials. Proc. Natl. Acad. Sci. U. S. A. 112, 11502 (2015).

50. F.W. Zok: On weakest link theory and Weibull statistics. J. Am. Ceram. Soc. 100, 1265 (2017).

51. W. Weibull: A statistical distribution function of wide applicability. J Appl Mech-T Asme. 18, 293 (1951).

52. W. Weibull: The Phenomenon of Rupture in Solids (Generalstabens Litografiska Anstalts Förlag, Stockholm, Sweden, 1939).

53. R.W. Hertzberg: Deformation and Fracture Mechanics of Engineering Materials (Wiley, New York, New York, 1976).

54. M.R. O'Masta, L. Dong, L. St-Pierre, H. Wadley, and V. Deshpande: The fracture toughness of octet-truss lattices. $J$. Mech. Phys. Solids 98, 271 (2017).

55. M.C. Messner: Optimal lattice-structured materials. J. Mech. Phys. Solids 96, 162 (2016)

56. I. Quintana-Alonso and N.A. Fleck: Fracture of brittle lattice materials: A review. In Major Accomplishments in Composite Materials and Sandwich Structures: An Anthology ONR Sponsored Research, I.M. Daniel, E.E. Gdoutos, Y.D.S. Rajapakse, eds. (Springer, Berlin, Germany, 2009); pp. 799-816.

57. Z.P. Bazant and S.D. Pang: Mechanics-based statistics of failure risk of quasibrittle structures and size effect on safety factors. Proc. Natl. Acad. Sci. U. S. A. 103, 9434 (2006).

58. M. Genet, G. Couegnat, A.P. Tomsia, and R.O. Ritchie: Scaling strength distributions in quasi-brittle materials from micro- to macro-scales: A computational approach to modeling nature-inspired structural ceramics. J. Mech. Phys. Solids $\mathbf{6 8}$, 93 (2014).

59. M.R. Begley, N.R. Philips, B.G. Compton, D.V. Wilbrink, R.O. Ritchie, and M. Utz: Micromechanical models to guide the development of synthetic 'brick and mortar' composites. J. Mech. Phys. Solids 60, 1545 (2012).

60. S-I. Roohani-Esfahani, P. Newman, and H. Zreiqat: Design and fabrication of 3D printed scaffolds with a mechanical strength comparable to cortical bone to repair large bone defects. Sci. Rep. 6, 19468 (2016)

\section{Supplementary Material}

To view supplementary material for this article, please visit https://doi.org/10.1557/jmr.2018.11. 